\title{
A feasibility study for the newborn screening of spinal muscular atrophy
}

Robert E. Pyatt, PhD, and Thomas W. Prior, PhD

\begin{abstract}
Purpose: The natural history of spinal muscular atrophy suggests that for maximum effect, therapeutics will need to be administered in the earliest phases of the disease. This will require the adoption of techniques for the genetic analysis of affected individuals at the newborn stage. Our objective was to examine the feasibility surrounding the newborn screening for spinal muscular atrophy. Methods: We investigated the application of real-time polymerase chain reaction technology for newborn screening. A multiplex assay was designed to identify homozygous deletions in SMN1 exon 7 and validated using 266 samples with defined SMN1 and SMN2 copy numbers. Sensitivity and specificity were then evaluated as part of a newborn screening strategy using DNA from 153 blood spots. Results: Real-time technology validation demonstrated correct exclusion of all normal and carrier samples, and identified the homozygous SMN1 exon 7 deletions in all 32 affected samples. In the series of blood spots, all 59 affected samples were correctly identified yielding an analytic sensitivity of 100\%; 56 normal and 39 carrier samples were correctly excluded yielding an analytic specificity of $100 \%$ for this blood spot series. Conclusion: We demonstrate that effective molecular technology exists and that ethics may soon warrant the newborn screening of spinal muscular atrophy. Genet Med 2006:8(7):428-437.
\end{abstract}

Key Words: newborn screening, spinal muscular atrophy, real-time PCR, SMN1

Spinal muscular atrophy (SMA) is the second most common autosomal recessive lethal condition in whites, ${ }^{1}$ with an incidence of approximately 1 in 6,000 to 10,000 live births. ${ }^{2}$ SMA is characterized by degeneration of anterior horn cells in the spinal cord and brain leading to progressive, proximal muscular weakness. ${ }^{1}$ On the basis of the age at presentation and clinical severity, childhood SMA has been classified into three subtypes. Type I (Online Mendelian Inheritance in Man [OMIM] 253300) is associated with an onset before 6 months of age, severe muscle weakness and hypotonia, an inability to sit, and respiratory failure that leads to death before the age of 2 years. $^{3-5}$ Muscle weakness in Type II (OMIM 253550) typically manifests between 6 and 12 months, and after 12 months in Type III (OMIM 253400). Type IV SMA (OMIM 271150) describes a form with adult onset of muscle weakness and features independent ambulation, a milder course, and normal lifespan.

SMA is caused by mutations in the survival motor neuron $(S M N)$ gene. ${ }^{6}$ The gene consists of nine exons and gives rise to the $38-\mathrm{kD}$ SMN protein, which functions in the assembly and regeneration of small nuclear ribonuclear proteins, ${ }^{7,8}$ premRNA splicing, ${ }^{9,10}$ and axonal RNA transport. ${ }^{11,12}$ The $S M N$ gene is located in an inverted, duplicated region of chromo-

From the Department of Pathology, Ohio State University, Columbus, Ohio. Thomas W. Prior, PhD, Department of Pathology, Ohio State University, Hamilton Hall 125, 1645 Neil Ave, Columbus, OH 43210.

Submitted for publication February 17, 2006.

Accepted for publication April 20, 2006.

DOI: 10.1097/01.gim.0000227970.60450.b2 some five containing two highly homologous copies of $S M N$, a telomeric copy (SMN1) and a centromeric copy (SMN2), ${ }^{6}$ which differ by five total bases including a single coding base pair $(840 \mathrm{C}>\mathrm{T}) .{ }^{13-15}$ The transcription of SMN1 results in fulllength mRNA in contrast with SMN2 transcription, which generates primarily a shortened mRNA species lacking exon 7 and a small amount of full-length transcript. ${ }^{16}$ This alteration in $S M N$ splicing is mediated by the exon 7 single-base change that distinguishes SMN1 from SMN2 and forms a site that preferentially binds the splice enhancer SF2/ASF to SMN1. ${ }^{17}$

Ninety-five percent of affected individuals have a homozygous deletion involving SMN1 exon 7 regardless of subtype. ${ }^{6,18}$ Although affected individuals retain at least one copy of SMN2, $S M N 2$ copy numbers vary in the unaffected population with $5 \%$ to $10 \%$ lacking both copies. ${ }^{6,19-21}$ The SMN2 gene is unable to compensate for the homozygous loss of SMN1 because of its alternative splicing, but SMN2 copy numbers appear to have a modifying effect on the variable SMA phenotype because of the small amount of full-length transcript produced..$^{22-25}$ Disease severity has been shown to correlate with SMN protein levels, ${ }^{23}$ and SMA Type I, II, or III has shown a significant correlation to SMN2 copy number. ${ }^{24,25}$ In one study, $96 \%$ of individuals with Type 1 carried one or two SMN2 copies, whereas $100 \%$ of individuals with Type III carried three or four copies. ${ }^{25}$

These findings suggest a potential therapeutic avenue for SMA: shifting the transcription of SMN2 from the alternatively spliced short form to more of the full-length species, which could compensate for the loss of SMN1. To date, the application of histone deacetylation inhibitors, including sodium butyrate, phenylbutyrate, valproic acid, hydroxyurea, and the 
chemotherapeutic agent aclarubicin, in cell culture and SMA animal models has demonstrated increased full-length SMN mRNA or protein..$^{26-31}$ These studies have met with enough success to warrant the examination of phenylbutyrate and valproic acid as therapeutic agents in clinical trials ${ }^{32}$ along with the ongoing development of gene-transfer methodologies. ${ }^{33,34}$ With the clinical course of SMA leading to the degeneration of the anterior horn motor neurons, the window of time for effective administration of these agents may be particularly short for affected individuals, especially those with the Type I phenotype. In a recent report examining motor nerve function and innervation in three infants with SMA Type I immediately from birth forward, the authors describe "severe and substantial postnatal progression of motor denervation ... with progression to generalized hypotonia and quadriparesis over a 1- to 2-week period." 35 Consequently, the timing for effective diagnosis and treatment, especially for the most severely affected individuals, may be short.

The molecular diagnosis of SMA has become a well-established practice with 28 laboratories currently participating in the College of American Pathologists SMA survey program. Individuals are typically referred for SMN1 deletion analysis on the basis of identification of disease-associated symptoms by a clinical physician. However, optimal intervention may require the administration of therapeutics while motor neurons are still present and the identification of individuals during the asymptomatic or presymptomatic phases for treatment. This could be potentially accomplished through a newborn screening program. SMA presents a unique challenge because such a strategy would require the use of DNA as a testing substrate and molecular techniques for deletion analysis, which differs from current practices.

In this report we present a feasibility study surrounding the adoption of newborn screening for SMA. We first investigated the sensitivity and specificity of real-time polymerase chain reaction (PCR) to scrutinize the application of this technology to newborn screening. A multiplex, real-time assay was designed to identify homozygous deletions in SMN1 using primers for $S M N$ exon 7 and a fluorescently labeled probe specific for the single-base change that defines $S M N 1$ along with a set of control primers and probe to RNase P. Validation of this assay for SMN1 exon 7 deletions was then conducted using 266 samples with known SMN1 and SMN2 copy numbers. Blood from 154 additional independent samples, including 39 carriers, 56 normal individuals, and 58 affected individuals, was spotted onto Guthrie cards. DNA was subsequently extracted and analyzed, demonstrating a high sensitivity and specificity for this technique. Finally, we consider the ethical issues and general economic cost surrounding the adoption of newborn screening for SMA using real-time PCR technology.

\section{MATERIALS AND METHODS}

All samples were sent to the Molecular Pathology Laboratory at Ohio State University for SMA mutation analysis or carrier testing, and informed consent was received for all sam- ples undergoing SMN analysis. All approved procedures for the handling of subjects were performed in compliance with the ethical standards of Ohio State University. For validation experiments of the real-time assay for homozygous SMN1 exon 7 deletions, DNA was extracted from whole blood using a standard salting-out procedure, and the concentration was determined with a spectrophotometer. ${ }^{36}$ A competitive, radiolabeled PCR reaction was performed on all samples to confirm $S M N 1$ deletions and determine SMN2 copy numbers as previously described (data not shown). ${ }^{37}$ For experiments to define the sensitivity and specificity of the real-time multiplex, $50 \mu \mathrm{L}$ of whole blood was spotted onto Guthrie cards and dried overnight at room temperature. DNA was extracted from blood spots using the Gentra Capture Card Kit (Gentra Systems, Minneapolis, $\mathrm{MN}$ ) into a volume of $50 \mu \mathrm{L}$ following the manufacturer's protocol. DNA concentrations were not calculated after extraction from blood spots. The remainder of the wholeblood sample was then extracted by salting out to simulate a recall blood sample for all first-tier positives. Deletion confirmation and determination of SMN2 copy numbers were again conducted using the competitive PCR and DNA from this extraction (data not shown). ${ }^{37}$

\section{Real-time polymerase chain reaction}

The SMN1 sequence specificity of the real-time reaction relies on the TaqMan probe because primers to exon 7 will rec-

Table 1

SMN1 and SMN2 copy numbers and total number of samples for each genotype used for validation of the real-time multiplex polymerase chain reaction

\begin{tabular}{lcc}
\hline SMN1 copy no. & SMN2 copy no. & No. of samples \\
\hline 0 & 3 & 1 \\
0 & 4 & 8 \\
0 & 5 & 21 \\
0 & 0 & 2 \\
1 & 1 & 5 \\
1 & 2 & 26 \\
1 & 3 & 34 \\
1 & 4 & 12 \\
1 & 5 & 5 \\
1 & 5 & 2 \\
2 & 0 & 5 \\
2 & 1 & 60 \\
2 & 2 & 72 \\
2 & 3 & 6 \\
3 & 0 & 1 \\
3 & 1 & 4 \\
4 & 0 & 1 \\
4 & 5 & 1 \\
\hline Total & 3 & \\
\hline
\end{tabular}


ognize both genes. We designed the 16 base pair, minor groove binding probe for SMN1 by using PrimerExpress software (Applied Biosystems, Branchburg, NJ) around the single-base change in exon 7 with the ninth base of the FAM-labeled probe at position 840 (5' CAG GGT TTC* AGA CAA A). We also included in each reaction an unlabeled, nonextending oligonucleotide specific for the base pair at position 840 in SMN2, which blocks the nonspecific interaction of the SMN1 probe to product from SMN2 (5' ATT TTC CTT ACA GGG TTT TAG ACA AAA TCA AAA GA-PHO 3').

Fluorescence is recorded over the course of the amplification program and is reported as a threshold cycle $\left(\mathrm{C}_{\mathrm{T}}\right)$ value that describes the fractional cycle at which point the fluorescence intensity passes a limit set within the exponential phase of the amplification curve. The differences in $\mathrm{C}_{\mathrm{T}}$ values can be used to measure the quantitative differences between two loci when primer sets to those sites amplify at similar efficiencies using a constant template concentration. Primers and a VIC-labeled probe for RNase P (Applied Biosystems) were included in each reaction as a control for quantification using the delta $\mathrm{C}_{\mathrm{T}}$ method in validation experiments only. In experiments on first-tier sensitivity and specificity, $R$ Nase $P$ serves as a control for successful DNA amplification because the lack of constant template concentrations in these reactions prevent the use of the delta $\mathrm{C}_{\mathrm{T}}$ calculation.

Real-time multiplex reactions were done in $25 \mu \mathrm{L}$ containing 1X TaqMan Universal PCR Master Mix without AmpErase UNG (Applied Biosystems), $900 \mathrm{nM} \mathrm{SMN1} \mathrm{exon} 7$ primers (F 5' ATA GCT ATT TTT TTT AAC TTC CTT TAT TTT CC $3^{\prime}$, R 5'CCT TAA TTT AAG GAA TGT GAG CAC C 3'), $250 \mu \mathrm{M}$ SMN1 MGB probe, $250 \mu \mathrm{M}$ SMN2 nonextending, competitive oligo, $1 \mathrm{X}$ RNase P control primers, and probe; $10 \mathrm{ng}$ of template was used for assay validation experiments, or $1 \mu \mathrm{L}$ of each blood spot extraction was used for tests of first-tier sensitivity and specificity. Reactions were carried out on an ABI Prism 7000 sequence detection system using 96-well MicroAmp optical plates (Applied Biosystems) and consisted of $50^{\circ} \mathrm{C}$ for 2 minutes, $95^{\circ} \mathrm{C}$ for 10 minutes, followed by 40 cycles of $95^{\circ} \mathrm{C}$ for 15 seconds and $60^{\circ} \mathrm{C}$ for 1 minute.

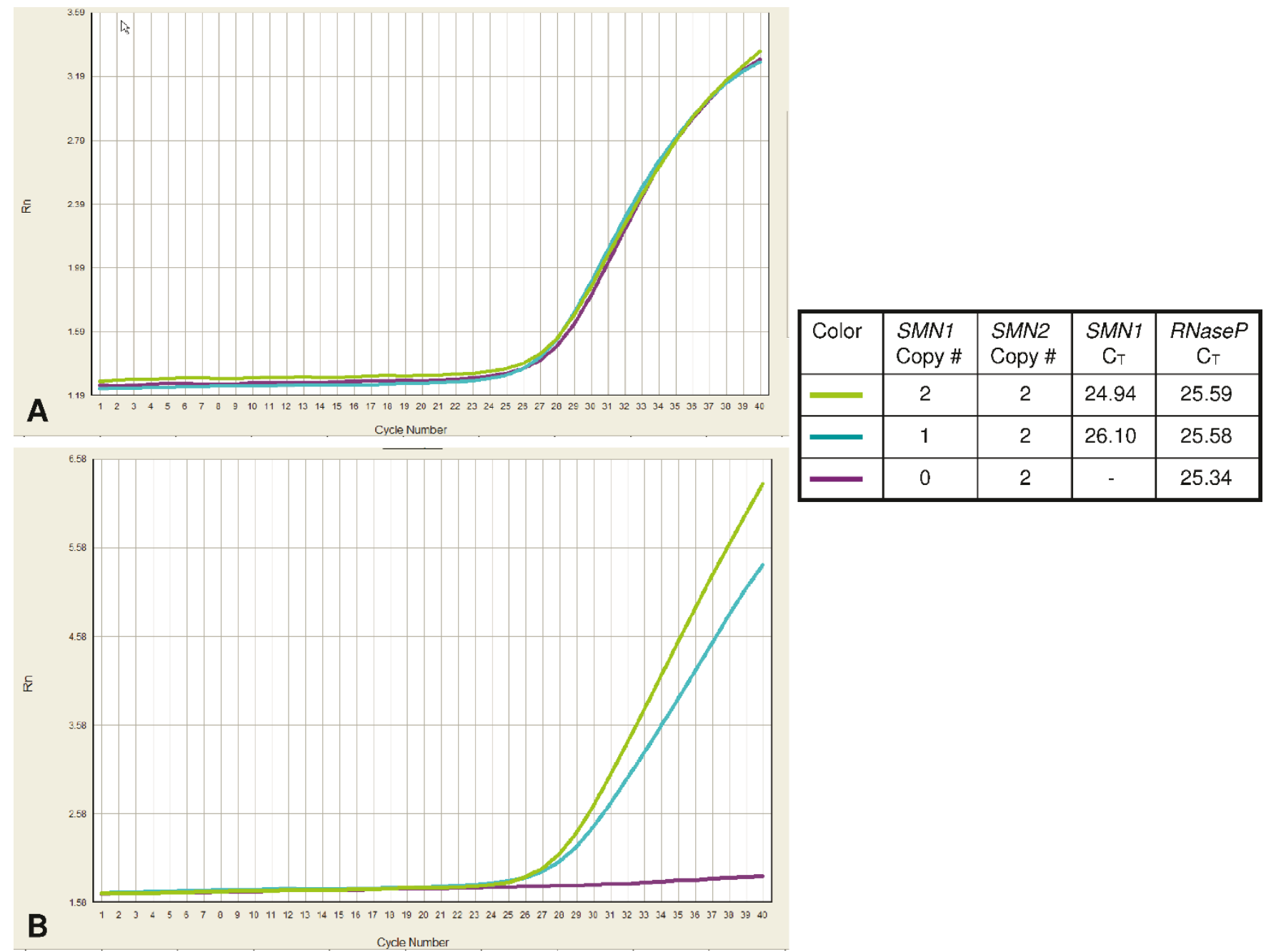

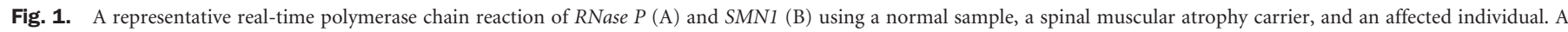

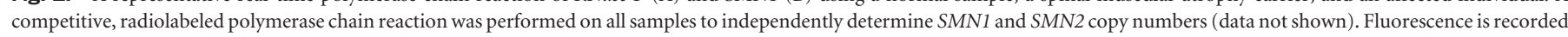

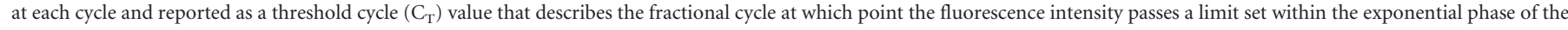
amplification curve. Gene copy numbers, corresponding amplification curve colors, and $\mathrm{C}_{\mathrm{T}}$ values (table at right). 


\section{RESULTS}

A total of 266 DNA samples of known SMN1 and SMN2 copy numbers (Table 1) were used to validate the detection of SMN1 exon 7 homozygous deletions by the multiplex real-time assay. Figure 1 shows a representative $S M N 1 /$ RNase P real-time multiplex amplification. Validation was performed with DNA extracted from whole blood, and a consistent $10 \mathrm{ng}$ of template was used in all reactions. All samples had previously confirmed SMN1 and SMN2 copy numbers (Table 1) and included 84 carriers and 32 individuals affected with SMA. With equal concentrations of template used in each reaction, $R$ Nase $P$ control $C_{\mathrm{T}}$ values are similar ranging from 25.34 to 25.59 (Fig. 1A), and RNase $P$ can be used to determine SMN1 copy numbers in each multiplex. In contrast, the normal sample (two-copy SMN1) had an SMN1 $\mathrm{C}_{\mathrm{T}}$ of 24.94 and the sample from the SMA carrier (one-copy SMN1) a $\mathrm{C}_{\mathrm{T}}$ value of approximately one cycle greater at 26.10 corresponding to one half the starting template (Fig. 1B). The sample from the affected individual (zero-copy SMN1) shows robust amplification at $R$ Nase $P$ (Fig. 1A) but fails to register fluorescent intensity from SMN1 at the threshold level (Fig. 1B). Similar results were obtained when the additional 31 affected individuals, 83 carriers, and 150 normal controls were analyzed.

We next evaluated the precision of the reaction for the detection of $S M N 1$ in the context of increasing copies of $S M N 2$ (Fig. 2). A control sample with two $S M N 1$ and two $S M N 2$ copies was amplified along with a series of affected individuals (zero-copy $S M N 1)$ with SMN2 copy numbers ranging from one to five. $R$ Nase $P$ values were again similar for all samples ranging from 25.70 to 25.02 (Fig. 2A) and the control two-copy SMN1 sample amplified at $S M N 1$ with a $C_{T}$ value of 25.42 . The affected samples show negligible amplification with residual levels only detected above background after 36 cycles or more (Fig. 2B). The background SMN1 levels detected in these affected samples can be attributed solely to the low-level interaction of the fluorescentlabeled SMN1 probe with product from the SMN2 gene. However, in all cases when samples with $S M N 2$ copies ranging from one to five were analyzed, there was no evidence of increasing

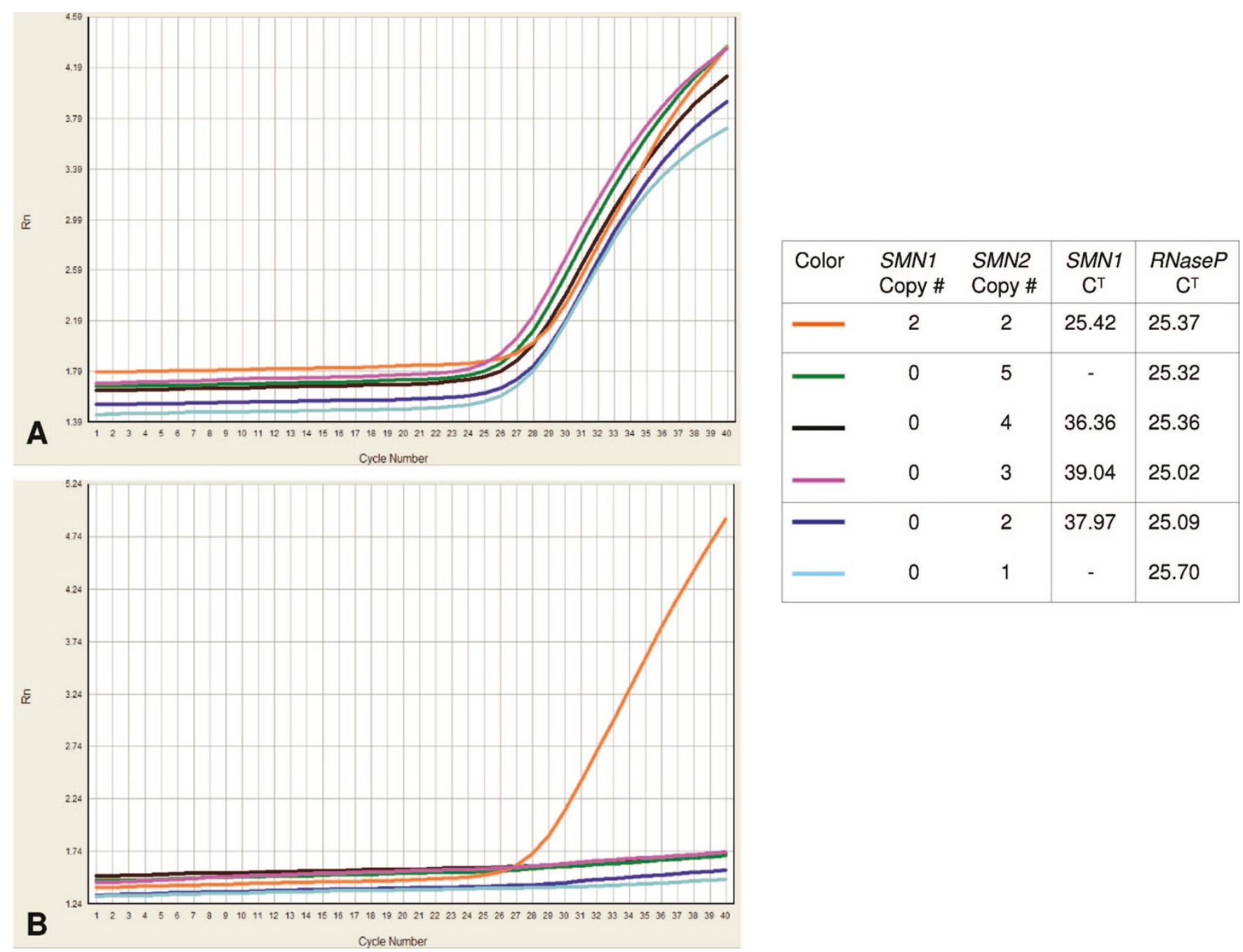

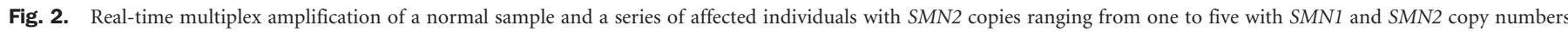

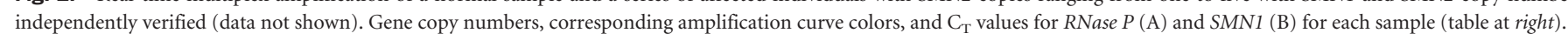


copies of SMN2 interfering with amplification from a two-copy SMN1 or carrier sample.

To examine the sensitivity and specificity of the real-time multiplex when applied to newborn screening, $50 \mu \mathrm{L}$ of whole blood from 153 additional independent samples (Table 2) were used to spot Guthrie cards simulating a newborn stick. Blood spots were extracted using the Gentra Capture card kit (Gentra Systems), and $1 \mu \mathrm{L}$ of each extraction was used in real-time multiplex amplifications. Without template quantification, $R$ Nase $P$ functions here as a control of DNA successful amplification from the blood spot extraction.

Representative analyses of blood spot samples are shown in Figures 3 and 4, and include an affected individual, an SMA carrier, and normal individuals (Fig. 3), and a series of affected individuals with SMN2 copy numbers ranging from 1 to 5 (Fig. 4). In both figures, all samples show amplification of $R$ Nase $P$ demonstrating the presence of amplifiable DNA from these blood spot extractions, although variations in amplification curves and $\mathrm{C}_{\mathrm{T}}$ values suggest that the concentration of DNA varies from sample to sample (Fig. $3 \mathrm{~A}$ and Fig. $4 \mathrm{~A}$ ). In addition, normal and carrier samples amplify at $S M N 1$ with $\mathrm{C}_{\mathrm{T}}$ values of 26.30 and 28.08 , respectively, whereas the affected sample fails to achieve amplification at the threshold level (Fig. $3 \mathrm{~B})$. Even with increasing copy numbers of $S M N 2$, affected samples do not reach the threshold level of amplification (Fig. $4 \mathrm{~B})$. Results were similar for all additional affected samples screened. The correct identification of all 57 affected samples gives this technology an analytic sensitivity of $100 \%$, and the correct exclusion of the 39 carrier and 56 normal samples also gives this strategy an analytic specificity of $100 \%$ for this blood spot series.

\section{Table 2}

SMN1 and SMN2 copy numbers and total number of samples for each genotype for blood spot samples analyzed with the real-time multiplex polymerase chain reaction assay

\begin{tabular}{lcc}
\hline SMN1 copy no. & SMN2 copy no. of samples \\
\hline 0 & 1 & 1 \\
0 & 2 & 22 \\
0 & 3 & 26 \\
0 & 4 & 9 \\
1 & 1 & 7 \\
1 & 2 & 25 \\
1 & 3 & 5 \\
1 & 4 & 2 \\
2 & 0 & 3 \\
2 & 1 & 18 \\
2 & 2 & 29 \\
2 & 3 & 3 \\
3 & 1 & 1 \\
3 & 2 & 2 \\
Total & & 153 \\
\hline
\end{tabular}

\section{DISCUSSION}

The classic Wilson and Jungner criteria ${ }^{38}$ for screening program evaluation can be reduced to three main questions: (1) Should we do it? (2) Can we do it? (3) Can we afford to do it?39 The adoption of newborn screening for any disorder requires that the benefits from an early diagnosis outweigh any potential harm caused by the screening process itself. Wilson and Jungner state that a condition screened for should be an "important health problem," 38 and the approximate incidence of 1 in 6,000 to 10,000 live births makes SMA a more common disorder than many of the diseases currently on newborn panels. The American College of Medical Genetics (ACMG) recently issued guidelines for evaluating newborn screening that downplayed the incidence of a particular disorder in favor of a more detailed examination of the benefits in testing for it. ${ }^{40}$

The benefits achieved through newborn screening have traditionally referred to the direct benefits to the affected child. Wilson and Jungner ${ }^{38}$ noted that that "there should be an acceptable treatment for patients with the recognized disease" and that "facilities for diagnosis and treatment should be available." Additional benefits from implementing a screening program may also include improvements in the quality of life and a decrease in early mortality. Newborn screening for cystic fibrosis $(\mathrm{CF})$ has led to a reduction in early infant mortality and improved nutrition, although it has not directly increased the life span of affected individuals. ${ }^{41,42}$ Although there are currently no effective treatments for SMA, a number of potential agents are currently being evaluated in clinical trials. ${ }^{32}$ Newly published work describing the natural history of the disease suggests that for therapeutics to be successful, they will need to be administered early during the course of the disease, before significant motor neuron degeneration has occurred. ${ }^{35}$ This may present a small window for effective therapeutic intervention, especially for the most severe form of the disease. This also presents a quandary for the ongoing trials that are evaluating the efficacy of agents in primarily symptomatic individuals rather than during the asymptomatic or presymptomatic phases of the disorder. Because these individuals have already experienced motor neuron degeneration, they may not represent the ideal population for the evaluation of an agent designed to improve motor neuron retention or prolong the onset of symptoms. It would seem more effective to develop a screening platform in parallel with therapeutic methods for SMA because the early identification of affected individuals would support the efficacy of the clinical trials, and the results from those trials are necessary for an accurate cost assessment of newborn screening adoption.

The recent ACMG task force for newborn screening also included benefits from early intervention to the family and to society in their evaluation of a disorder's suitability for inclusion. ${ }^{40} \mathrm{An}$ accurate and early diagnosis, particularly for an often misdiagnosed disorder, can provide closure and comfort for parents, and prevent additional, unnecessary investigations..$^{39,40,43}$ The genetic information obtained through newborn screening is also relevant to the child's family concerning the prevention of 


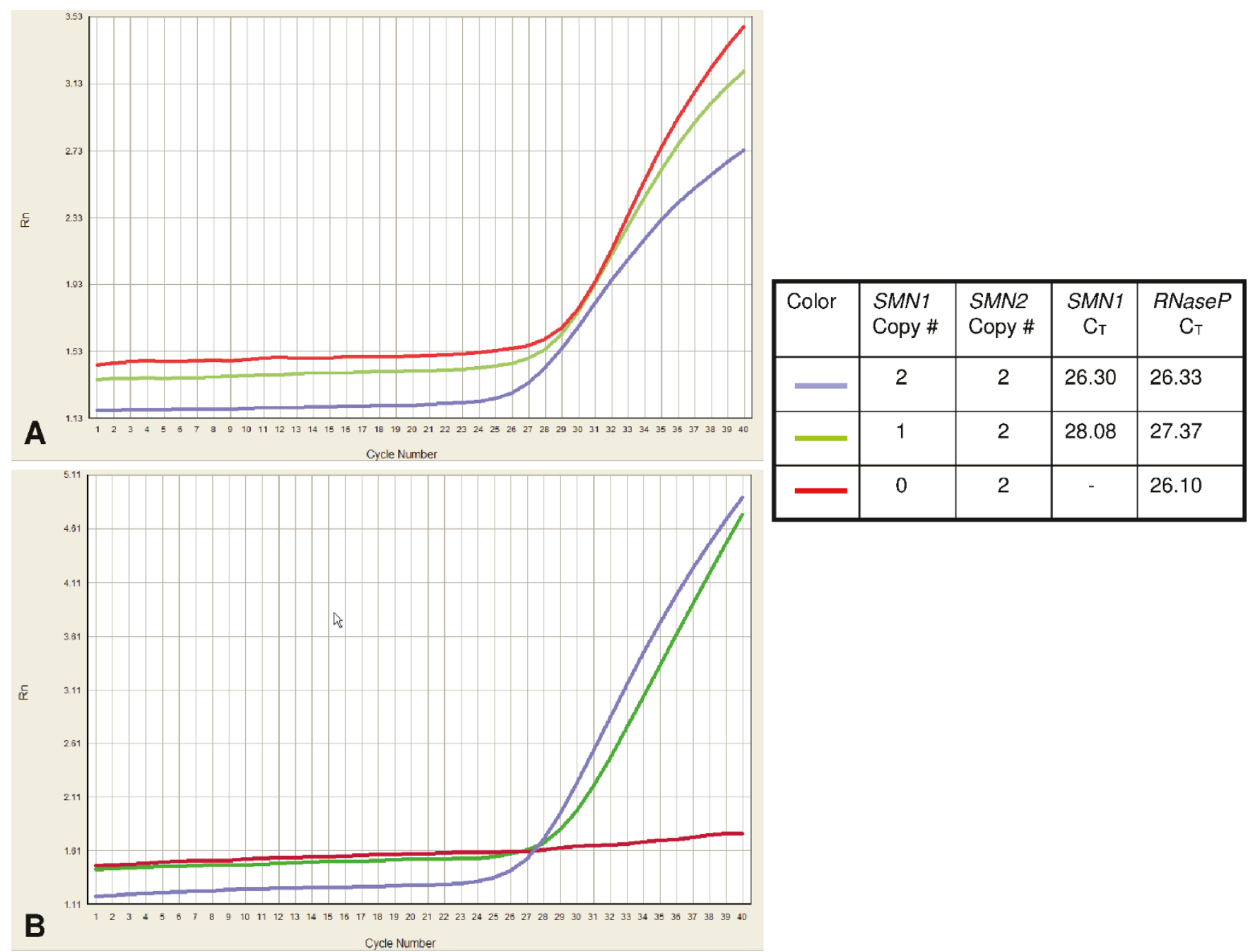

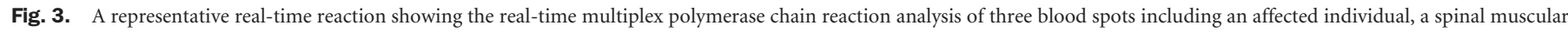

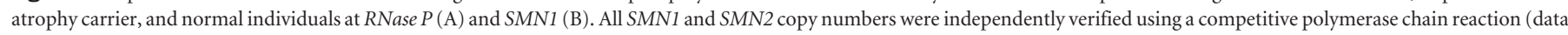
not shown, see "Materials and Methods"). Gene copy numbers, corresponding amplification curve colors, and $\mathrm{C}_{\mathrm{T}}$ values for each sample (table at right).

additional cases through genetic counseling and the carrier testing of siblings and other extended relatives. SMA also presents a unique case in which prognostic information regarding disease severity in affected individuals is also available as a further benefit to families. There are significant data supporting the modification of the SMA phenotype by SMN2 copy number with the presence of three or more SMN2 copies correlated with a milder disease phenotype. ${ }^{22-25}$ Any information of predictive value regarding the severity of a child's illness will obviously be of interest to parents and can be collected along with deletion confirmation in the second tier of the screening strategy outlined here. Reporting of these results should be conducted with the greatest of care using appropriate genetic counselors because there are rare cases of individuals with SMA Type I with three SMN2 copies. Newborn screening for SMA would also provide invaluable scientific information, such as the true incidence of the disease and any differences in frequency seen between the major racial groups. In this light, the emphasis of newborn screening can involve more than the medical care of the affected child alone.
"Can we do it?" asks if the technology and technical expertise exist to precisely identify affected cases on a large scale. Unlike CF, in which elevated immunoreactive trypsinogen levels are diagnostic for the disease, SMA has no characteristic biochemical phenotype and diagnosis must be done at the DNA level. ${ }^{44}$ Any newborn screening strategy for SMA would require DNA extraction from blood spots followed by a twotiered strategy using DNA as a testing substrate at both levels as depicted in Figure 5. Although some states use genetic analysis for confirmatory testing of samples with elevated immunoreactive trypsinogen levels suggestive of $\mathrm{CF},{ }^{44}$ there are currently no newborn screening programs in the United States that use DNA as a first-tier substrate. The implementation of newborn screening for SMA would then require the adoption of new technologies in the screening laboratories both in terms of handling a new substrate for first-tier testing and new molecular technologies for that analysis.

By using Ohio as an example, newborn screening for SMA would first require a high-throughput methodology for the DNA extraction from the 400 to 500 blood spots typically re- 


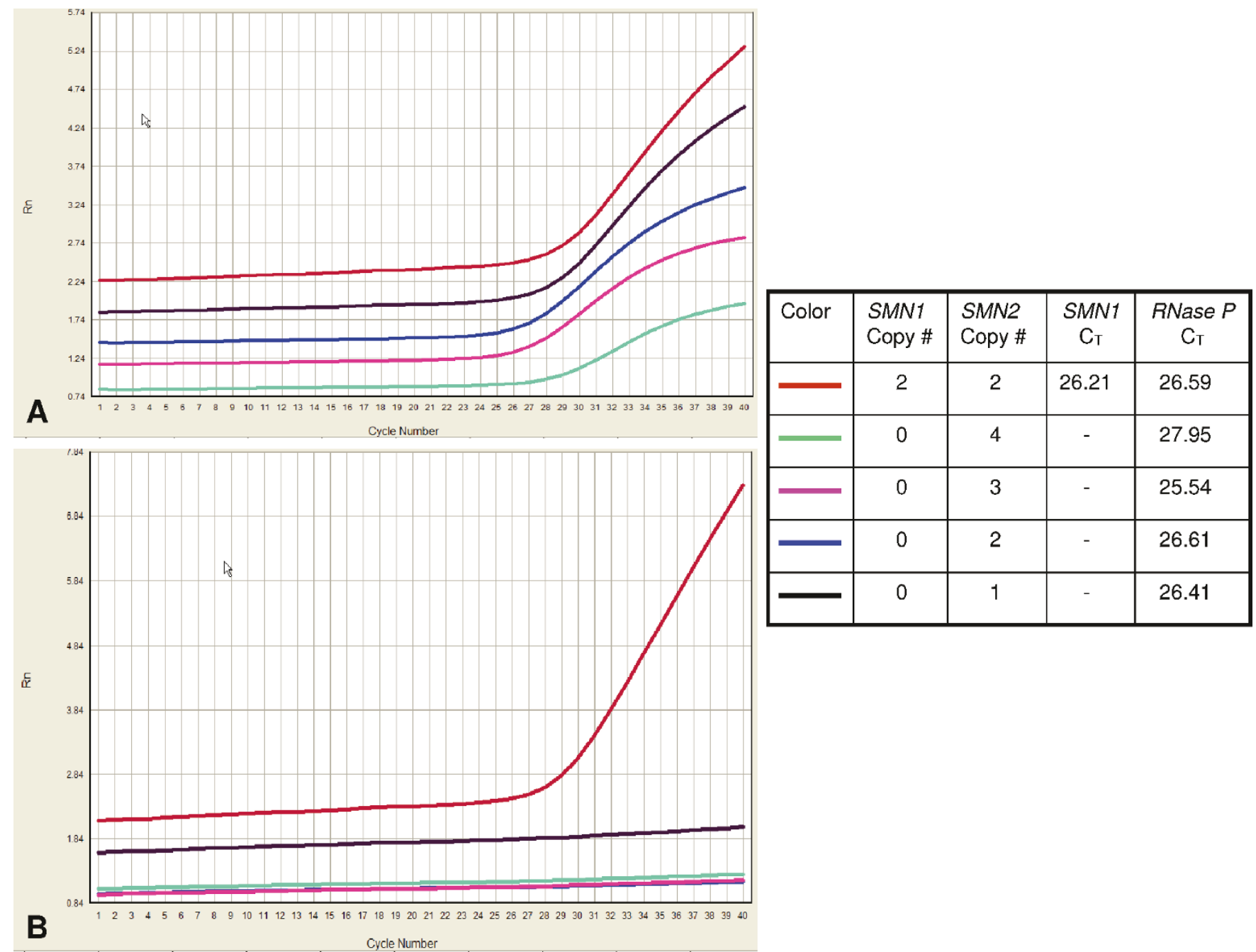

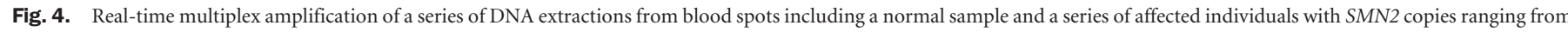

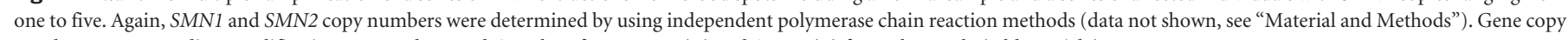
numbers, corresponding amplification curve colors, and $\mathrm{C}_{\mathrm{T}}$ values for RNase P (A) and SMN1 (B) for each sample (table at right).

ceived daily at the State Department of Health Laboratory. This methodology would need to use a rapid chemistry, be open to automation for high throughput, and produce DNA of a quality appropriate for the technique used in the first-tier analysis. Although the extractions in this report were not automated, we chose the Gentra chemistry because this technique uses a simple procedure to produce DNA in a timeefficient manner that is suitable for PCR amplification. In addition, the Gentra chemistry can be automated for use on a liquid handling system and achieve the high throughput ultimately needed. ${ }^{45}$

The first tier of a screening program for SMA would also require a high-throughput methodology open to automation for the detection of affected samples (Fig. 5). The real-time PCR technique described in this report requires approximately 100 minutes to amplify a 96-well plate typically followed by less than 1 hour for interpretation. In a series of 154 blood spots, the real-time multiplex PCR demonstrated an analytic sensitivity and specificity of $100 \%$ correctly identifying 59 affected samples and excluding 92 normal individuals or carriers. Following the model currently used by many of the state laboratories conducting CF screening, primary care physicians would be contacted and notified of results for all first-tier positive samples to obtain recall samples of fresh blood for confirmation using the second-tier technique. ${ }^{44}$ Because significantly fewer samples would be analyzed using the second-tier methodology, procedures at this level would not have the same throughput or automation requirements as those of the first tier and could use previously established methods. As outlined in Figure 5, this could be accomplished with a competitive PCR previously developed in our laboratory that is currently used in the molecular diagnosis of SMA. ${ }^{37}$ This would provide independent confirmation of SMN1 exon 7 homozygous deletions using an independent method from the first tier and simultaneously quantify SMN2 levels.

Although this newborn screening strategy does exhibit high analytic sensitivity in the identification of affected individuals with homozygous deletions in SMN1 exon 7, it will not identify 


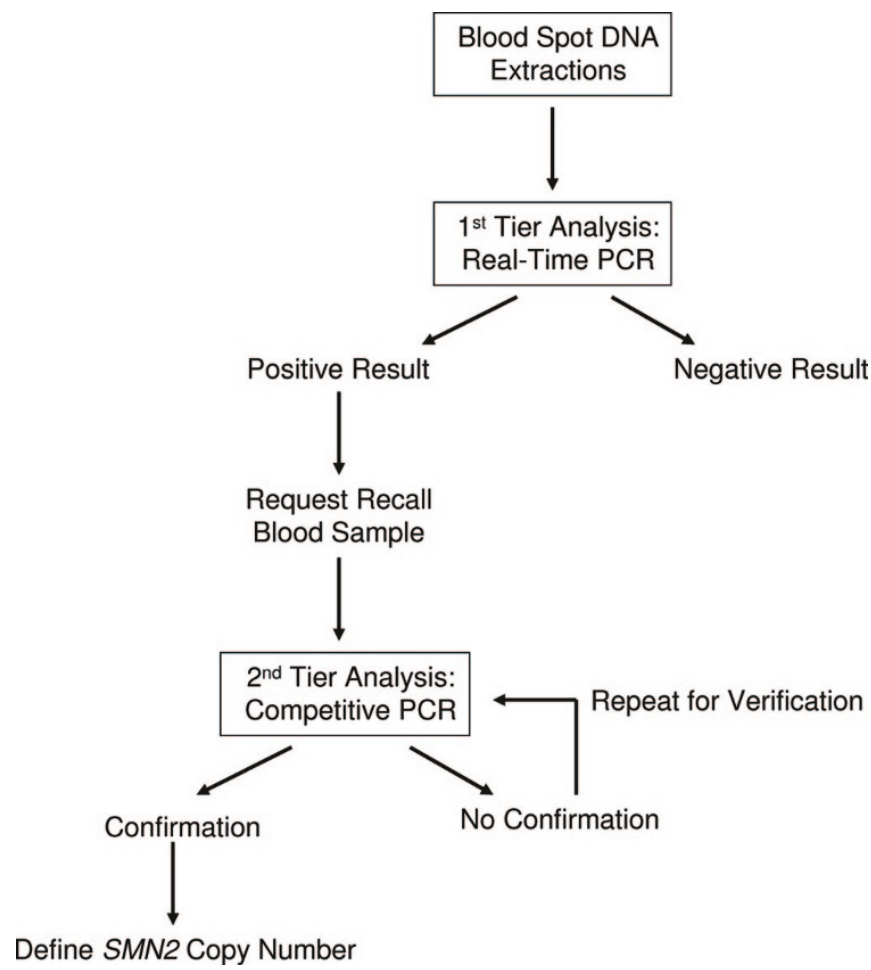

Fig. 5. The proposed two-tiered strategy for newborn screening of spinal muscular atrophy (SMA) using DNA as a testing substrate at both levels. All blood spots would undergo extraction and first-tier analysis with the real-time polymerase chain reaction (PCR) protocol outlined in this report. A positive result from the first tier would trigger the collection of a recall sample of whole blood through the individual's primary care physician and subsequent analysis using the competitive PCR methodology currently used for SMA molecular analysis. This procedure would both confirm the homozygous deletion in SMN1 and quantify SMN2 gene copy numbers. Recall samples not confirming a positive result from the first tier would be reanalyzed to confirm the negative result using the competitive PCR.

the approximate $5 \%$ of affected individuals who are compound heterozygotes possessing one deleted $S M N 1$ allele and a second allele with a point mutation. The overall sensitivity of this methodology is approximately $95 \%$. Correct exclusion was also made for all carrier and normal samples in our blood spot series; however, there have been rare cases of asymptomatic individuals with homozygous SMN1 deletions. The series of blood spots used for evaluation did not contain any samples with this genotype, but the DNA samples used to validate the real-time PCR assay contained two samples with zero SMN1 copies and five SMN2 copies, both from previously reported phenotypically normal individuals..$^{20}$ The exact frequency of asymptomatic carriers with homozygous deletions in the general population is not known, but these individuals do seem to be rare, having only been identified through family studies with affected probands and not seen in more than 700 normal control individuals with no history of SMA in our laboratory. The presence of these rare false-positives would necessitate an estimated clinical specificity of more than $99 \%$ for the SMA newborn screening strategy.

The potential harm and nontechnical costs inherent in both questions of "Should we do it?" and "Can we do it?" also need to be addressed. Although unaffected individuals with ho- mozygous deletions in SMN1 exon 7 and high SMN2 copy numbers are rare, their exact frequency in the population is currently unknown. This misdiagnosis could cause unnecessary apprehension to parents along with possible side effects from treating unaffected children with SMA-specific therapeutic agents. The second tier of the newborn screening strategy outlined in this article yields an independent confirmation of the SMN1 exon 7 homozygous deletion and a quantification of the SMN2 copy number. Because of the modifying effect of increased SMN2 copy number on the disease phenotype, the addition of this information as a part of the screening platform would provide valuable information on disease severity to these families.

The misdiagnosis of affected cases is also of concern because these individuals would need to be identified later in the symptomatic phase through clinical diagnosis on the basis of the first expression of muscle weakness, generalized hypotonia, or other symptoms. The approximate $5 \%$ of patients who are compound heterozygotes would be in this category, and how the timing of this later-phase diagnosis would overlap with the window for effective treatment is unknown. The families of these children would certainly feel an emotional impact and express a negative reaction toward this misdiagnosis and its potential impact on their child's quality of life. Addressing the identification of compound heterozygotes is something of a double-edged sword because any technology sensitive enough to detect individuals with a single deleted copy of SMN1 would also identify all carriers for the disease with a frequency of 1 in 40 to 1 in 60. Unwanted carrier detection in newborn screening is an issue because of the potential violation of the child's ethical right to autonomy and has been particularly encountered in the context of screening for CF. ${ }^{46}$ The real-time PCR assay outlined in this report was specifically designed to exclusively identify only homozygous SMN1 deletions and preclude the unwanted identification of SMA carriers.

Finally, the question "Can we afford to do it?" directly addresses the technical cost of implementing large-scale DNA screening. Tandem mass spectroscopy, the primary technique currently used in most first-tier screening programs, is ideal because it is a relatively inexpensive technology with minimal incremental costs for additional tests. The recent ACMG recommendations support the use of multiplex technologies in newborn screening for those same reasons. Unfortunately, most technologies currently available for genetic analysis do not feature the required throughput, expansion capability, or per sample cost necessary for their use in newborn screening. Although we have shown that real-time technology has a high sensitivity and specificity in application to SMA, it has a relatively expensive per sample cost and limited expansion capabilities for multiplexing additional tests and disorders. Accurate health economic assessment requires data, such as the cost estimate per prevented death and cost estimate per preventable impairment, that are not currently available for SMA.

We have demonstrated that newborn screening for SMA can be technically accomplished. We have presented a two-tiered newborn screening strategy using DNA at both levels and dem- 
onstrated that the described real-time multiplex PCR has the sensitivity and specificity necessary for application to the first tier. With strong support from advocacy groups for implementing such screening, the next step would be the development of a pilot program offering voluntary testing and using informed consent procedures that would gather the necessary data for accurate assessment of SMA newborn screening. Such a program would provide important information on the incidence of the disorder on the population level; parental attitudes, reactions, and concerns; evaluation of screening technology using the analysis of true population samples; and accurate economic assessment. In addition, such a pilot program would allow for the immediate enrollment of affected children into ongoing clinical trials to allow for administration of therapies during the critical treatment phase of the disease and consequently the more accurate examination of their effectiveness.

\section{ACKNOWLEDGMENT}

We recognize the Families of SMA for their support and involvement in this work. This research was supported by the National Institutes of Health grant 174615.

\section{References}

1. Crawford TO, Pardo CA. The neurobiology of childhood spinal muscular atrophy. Neurobiol Dis 1996;3:97-110.

2. Pearn J. Genetic studies of acute infantile spinal muscular atrophy (SMA type I). An analysis of sex ratios, segregation ratios, and sex influence. J Med Genet 1978;15:414417.

3. Pearn J. Classification of spinal muscular atrophies. Lancet 1980;1:919-922.

4. Dubowitz V. Chaos in the classification of SMA: a possible resolution. Neuromuscul Disord 1995;5:3-5.

5. Zerres K, Davies KE. 59th ENMC International Workshop: spinal muscular atrophies: recent progress and revised diagnostic criteria 17-19 April 1998. Soestduinen, The Netherlands. Neuromuscul Disord 199;9:272-278.

6. Lefebvre S, Burglen L, Reboullet S, Clermont O, et al. Identification and characterization of a spinal muscular atrophy-determining gene. Cell 1995;80:155165 .

7. Meister G, Fischer U. Assisted RNP assembly: SMN and PRMT5 complexes cooperate in the formation of spliceosomal UsnRNPs. EMBO J 2002;21:58535863.

8. Paushkin S, Gubitz AK, Massenet S, Dreyfuss G, et al. The SMN complex, an assemblyosome of ribonucleoproteins. Curr Opin Cell Biol 2002;14:305-312.

9. Pellizzoni L, Baccon J, Rappsilber J, Mann M, et al. Purification of native survival of motor neurons complexes and identification of Gemin6 as a novel component. $J$ Biol Chem 2002;277:7540-7547.

10. Gubitz AK, Mourelatos Z, Abel L, Rappsilber J, et al. A novel WD repeat protein component of the SMN complex that binds Sm proteins. J Biol Chem 2002;277: 5631-5636.

11. Bechade C, Rostaing P, Cisterni C, Kalisch R, et al. Subcellular distribution of survival motor neuron (SMN) protein: possible involvement in nucleocytoplasmic and dendritic transport. Eur J Neurosci 1999;11:293-304.

12. Pagliardini S, Giavazzi A, Setola V, Lizier C, et al. Subcellular localization and axonal transport of the survival motor neuron (SMN) protein in the developing rat spinal cord. Hum Mol Genet 2000;9:47-56.

13. Lorson CL, Hahnen E, Androphy EJ, Wirth B, et al. A single nucleotide in the SMN gene regulates splicing and is responsible for spinal muscular atrophy. Proc Natl Acad Sci U S A 1999;96:6307-6311.

14. Lorson CL, Androphy EJ. An exonic enhancer is required for inclusion of an essential exon in the SMA determining gene SMN. Hum Mol Genet 2000;9:259265.

15. Cartegni L, Krainer AR. Disruption of an SF2/ASF-dependent exonic splicing enhancer in SMN2 causes spinal muscular atrophy in the absence of SMN1. Nat Genet 2002;30:377-384.
16. Monani UR, Lorson CL, Parsons DW, Prior TW, et al. A single nucleotide difference that alters splicing patterns distinguishes the SMA gene SMN1 from the copy gene SMN2. Hum Mol Genet 1999;8:1177-1183.

17. Cartegni L, Hastings Ml, Calarco JA, de Stanchina E, et al. Determinants of exon 7 splicing in the Spinal Muscular Atrophy Genes, SMN1 and SMN2. Am J Hum Genet 2006;78:63-77.

18. Wirth B. An update of the mutation spectrum of the survival motor neuron gene (SMN1) in autosomal recessive spinal muscular atrophy (SMA). Hum Mutat 2000; 15:228-237.

19. McAndrew PE, Parsons DW, Simard LR, Rochette C, et al. Identification of proximal spinal muscular atrophy carriers and patients by analysis of SMNT and SMNC gene copy number. Am J Hum Genet 1997;60:1411-1422.

20. Prior TW, Swoboda KJ, Scott HD, Hejmanowski AQ, et al. Homozygous SMN1 deletions in unaffected family members and modification of the phenotype of SMN2. Am J Med Genet 2004;130A:307-310.

21. Gerard B, Ginet N, Matthijs G, Evrard P, et al. Genotype determination at the survival motor neuron locus in a normal population and SMA carriers using a competitive PCR and primer extension. Hum Mutat 2000;16:253-263.

22. Velasco E, Valero C, Valero A, Moreno F, et al. Molecular analysis of the SMN and NAIP genes in Spanish spinal muscular atrophy (SMA) families and correlation with number if copies of cBCD541 and SMA phenotype. Hum Mol Genet 1996;5:257263.

23. Lefebvre S, Burlet P, Liu Q, Bertrandy S, et al. Correlation between severity and SMN protein level in spinal muscular atrophy. Nat Genet 1997;16:265-269.

24. Feldkotter M, Schwarzer V, Wirth R, Wienker TF, et al. Quantitative analyses of SMN1 and SMN2 based on real-time lightcycler PCR: fast and highly reliable carrier testing and prediction of severity of spinal muscular atrophy. Am J Hum Genet 2002;70:358-368.

25. Mailman MD, Heinz JW, Papp AC, Snyder PJ, et al. Molecular analysis of spinal muscular atrophy and modification of the phenotype by SMN2. Genet Med 2002;4: 20-26.

26. Chang JG, Hsieh-Li HM, Jong YJ, Wang NM, et al. Treatment of spinal muscular atrophy by sodium butyrate. Proc Natl Acad Sci U S A 2001;98:9808-9813.

27. Andreassi C, Angelozzi C, Tiziano FD, Vitali T, et al. Phenylbutyrate increases SMN expression in vitro: relevance for treatment of spinal muscular atrophy. Eur J Hum Genet 2004;12:59-65.

28. Andreassi C, Jarecki J, Zhou J, Coovert DD, et al. Aclarubicin treatment restores SMN levels to cells derived from type I spinal muscular atrophy patients. Hum $\mathrm{Mol}$ Genet 2001;10:2841-2849.

29. Sumner CJ, Huynh TN, Markowitz JA, Perhac JS, et al. Valproic acid increases SMN levels in spinal muscular atrophy patient cells. Ann Neurol 2003;54:647654.

30. Brichta L, Hofmann Y, Hahnen E, Siebzehnrubl FA, et al. Valproic acid increases SMN2 protein level: a well known drug as potential therapy for spinal muscular atrophy. Hum Mol Genet 2003;12:2481-2489.

31. Grzeschik SM, Ganta M, Prior TW, Heavlin WD, et al. Hydroxyurea enhances SMN2 gene expression in spinal muscular atrophy cells. Ann Neurol 2005;58:194202.

32. Mercuri E, Bertini E, Messina S, Pelliccioni M, et al. Pilot trial of phenylbutyrate in spinal muscular atrophy. Neuromuscul Disord 2004;14:130-135.

33. DiDonato CJ, Parks RJ, Kothary R. Development of a gene therapy strategy for the restoration of survival motor neuron protein expression: implications for spinal muscular atrophy therapy. Hum Gene Ther 2003;14:179-188.

34. Lesbordes JC, Cifuentes-Diaz C, Miroglio A, Joshi V, et al. Therapeutic benefits of cardiotrophin-1 gene transfer in a mouse model of spinal muscular atrophy. Hum Mol Genet 2003;12:1233-1239.

35. Swoboda KJ, Prior TW, Scott CB, McNaught TP, et al. Natural history of denervation in SMA: relation to age, SMN2 copy number, and function. Ann Neurol 2005; 57:704-712.

36. Miller SA, Dykes DD, Polesky HF. A simple salting out procedure for extracting DNA from human nucleated cells. Nucleic Acids Res 1988;16:1215.

37. McAndrew PE, Parsons DW, Simard LR, Rochette C, et al. Identification of proximal spinal muscular atrophy carriers and patients by analysis of $\mathrm{SMN}^{\mathrm{T}}$ and $\mathrm{SMN}^{\mathrm{C}}$ gene copy number. Am J Hum Genet 1997;60:1411-1422.

38. Wilson JMG, Jungner G. Principals and practices of screening for disease. Geneva: World Health Organization 1968.

39. Wilcken B. Ethical issues in newborn screening and the impact of new technologies. Eur J Pediatr 2003;162:S62-S66.

40. Newborn screening toward a uniform screening panel and system. Rockville, MD: Maternal and Child Health Bureau, March 2005. http://mchb.hrsa.gov/screening/. Accessed 1 April 2006.

41. Farrell PM, Kosorok MR, Rock MJ, Laxova A, et al. Early diagnosis of cystic fibrosis through neonatal screening prevents sever malnutrition and improves long-term growth. Pediatrics 2001;107:1-13. 
42. Wilcken B, Chalmers G. Reduced morbidity in cystic fibrosis. Lancet 1986;1:439.

43. Walter JH. Arguments for early screening: a clinician's perspective. Eur J Pediatr 2003;162:S2-S4.

44. Wilfond BS, Gollust SE. Policy issues for expanding newborn screening programs: the cystic fibrosis newborn screening experience in the United States. J Pediatr 2005; 146:668-674.
45. Heath EM, O'Brien DP, Banas R, Naylor EW, et al. Optimization of an automated DNA purification protocol for neonatal screening. Arch Pathol Lab Med 1999;123: 1154-1160.

46. Wilcken B, Wiley V, Sherry G, Bayliss U, et al. Neonatal screening for cystic fibrosis: a comparison of two strategies for case detection in 1.2 million babies. J Pediatr 1995;127:965-970. 